\title{
Activity and functional diversity of microbial communities in long-term hydrocarbon and heavy metal contaminated soils
}

\author{
Anna Markowicz ${ }^{1 *}$, Grażyna Płaza ${ }^{2}$, Zofia Piotrowska-Seget ${ }^{1}$ \\ ${ }^{1}$ University of Silesia, Poland \\ Department of Microbiology \\ ${ }^{2}$ Institute for Ecology of Industrial Areas, Poland \\ *Corresponding author's e-mail: anna.markowicz@us.edu.pl
}

\begin{abstract}
Keywords: polycyclic aromatic hydrocarbons, heavy metals, microbial activity, functional diversity, Community Level Physiological Profiles.

Abstract: The impacts of long-term polycyclic aromatic hydrocarbons (PAHs) and heavy metal pollution on soil microbial communities functioning were studied in soils taken from an old coke plant. The concentrations of PAHs in the tested soils ranged from 171 to $2137 \mathrm{mg} \mathrm{kg}^{-1}$. From the group of tested heavy metals, concentrations of lead were found to be the highest, ranging from 57 to $3478 \mathrm{mg} \mathrm{kg}^{-1}$, while zinc concentrations varied from 247 to $704 \mathrm{mg} \mathrm{kg}^{-1}$ and nickel from 10 to $666 \mathrm{mg} \mathrm{kg}^{-1}$. High dehydrogenase, acid and alkaline phosphatase activities were observed in the most contaminated soil. This may indicate bacterial adaptation to long-term heavy metal and hydrocarbon contamination. However, the Community Level Physiological Profiles (CLPPs) analysis showed that the microbial functional diversity was reduced and influenced to a higher extent by some metals $(\mathrm{Pb}, \mathrm{Ni})$, moisture and conductivity than by PAHs.
\end{abstract}

\section{Introduction}

Due to progressive industrialization polycyclic aromatic hydrocarbons (PAHs) and heavy metals have been excessively released into the environment thus creating a major global concern. PAHs are ubiquitous contaminants known for their carcinogenic, mutagenic and teratogenic properties (Wang et al. 2010, Veses et al. 2013, Rachwał et al. 2015). These hydrophobic compounds are among the persistent organic pollutants, some of them are listed as US-EPA and European priority contaminants. They reveal high solid-water distribution ratio, low aqueous solubility and low bioavailability (Bamforth and Singleton 2005, Lu et al. 2011). In soils, PAHs diffuse into nanopores of soil particles and bind into organic matter. This natural process, called ageing, strongly affects biological toxicity and biodegradability of hydrocarbons (Tang et al. 2012, Cébron et al. 2013). Hydrocarbon contaminants often co-exist with heavy metals, which is the effect of their common source of pollution. Similarly to PAHs heavy metals, present at elevated concentrations, might decrease the number of soil microorganisms, their activity and biodiversity (Klimek and Niklińska 2007, Lu et al. 2013). It has been reported that the combined effects of organic and metallic contaminants on soil biology may be more toxic than those of PAHs alone (Thavamani et al. 2012, Lu et al. 2013). Since the bioremediation relies on the degradation of hydrocarbons by soil microorganism the estimation of the impact of long- term mixed pollution on the activity and functional diversity of microbial communities has considerable benefits for risk assessment and remediation strategies. The understanding of how autochthonic microbial populations respond to mixed contamination present at various concentrations is still limited. Therefore, the aims of the present study were to determine (i) the chemical profile of PAHs and heavy metals in long-term contaminated soils, (ii) the effects of long-term organic and metallic co-contamination on soil microbial activity by assessing the activity of several soil enzymes (dehydrogenase, urease, acid and alkaline phosphatase), and the functional diversity using the Biolog method.

\section{Materials and methods}

\section{Site description and soil sampling}

The contaminated soil samples used in this study were collected from a 120-year-old coke oven plant located in Upper Silesia, Poland. Coke production at this site began in 1884 . In preliminary investigations concentration of $\mathrm{PAH}$ and heavy metal in several samples taken from the coke oven territory and the adjacent area $\left(50^{\circ} 19 \mathrm{~N}, 18^{\circ} 47 \mathrm{E}\right)$ were determined. These analyses revealed that PAHs contamination was unevenly distributed across the tested area. On the basis of different levels of PAH and heavy metal contamination four sample subareas were selected. The soil samples were taken from the upper horizon ( $0-20 \mathrm{~cm}$ depth), and each soil sample was 
a composite of 5 mixed and homogenized subsamples collected at the four corners and the centre of an area of $10 \times 10 \mathrm{~m}$. The samples were air-dried and sieved (mesh size $2 \times 2 \mathrm{~mm}$ ) to remove the coarse fraction.

\section{Determination of the soil properties}

The physicochemical properties: $\mathrm{pH}$ in water, conductivity, soil total N, P and soil particle size distribution (size classification according to PN-R-04033 1998) were determined using the ISO standards (ISO 11263 1994, ISO 11261 1995, ISO 10390 1997, ISO 11265 1997, ISO 11277 1998). Organic matter (OM) content was determined by sulfochromic oxidation of organic carbon followed by titration of the excess $\mathrm{K}_{2} \mathrm{Cr}_{2} \mathrm{O}_{7}$ with $\mathrm{FeSO}_{4}\left(\mathrm{NH}_{4}\right)_{2} \mathrm{SO}_{4} \cdot 6 \mathrm{H}_{2} \mathrm{O}$ as described by Schinner et al. (1996). The 16 EPA priority PAHs in contaminated soils were identified and determined according to the ISO (ISO 13877 1998) and the EPA Standard Methods (USEPA 1992, 1995). PAHs were detected by high-performance liquid chromatography coupled with a fluorescence detector (HPLC-FLD Agilent 1200). Heavy metal contents (Cd, Pb, Ni, $\mathrm{Zn}, \mathrm{Cu}$ ) were determined after mineralization with aqua regia using atomic absorption spectrometry (SpectrAA 300 Varian) with graphite furnace. The limit of detection and recovery values of tested parameters are presented in Table 1.

\section{Enzyme assays}

The microbial activities, in terms of soil enzymes, were measured using the following methods. Dehydrogenase activity (DHA) was determined using triphenyltetrazolium chloride as an artificial electron acceptor and expressed as $\mu \mathrm{g}$ triphenyl formazone (TPF) $\mathrm{g}^{-1}$ dry soil $24 \mathrm{~h}^{-1}$ (Alef 1995). Soil urease activity was expressed as $\mu \mathrm{g} \mathrm{N}-\mathrm{NH}_{4} \mathrm{~g}^{-1}$ dry soil $\mathrm{h}^{-1}$ (Tabatabai and Bremner 1972). The activities of alkaline and acid phosphatases were expressed as $\mathrm{mg} p$-nitrophenol $\mathrm{g}^{-1}$ dry soil h $\mathrm{h}^{-1}$ (Tabatabai and Bremner 1969).

\section{Community-level physiological profiles (CLPPs)}

Community level physiological profiles (CLPPs) were assessed using the Biolog EcoPlate ${ }^{\mathrm{TM}}$ system (Biolog Inc., CA, USA). Briefly, microorganisms were extracted from soil suspensions ( $10 \mathrm{~g}$ dry weight of soil in $90 \mathrm{ml}$ sterile $0.85 \% \mathrm{NaCl}$ solution) and aliquots of $125 \mu \mathrm{l}$ were inoculated on plates, which were incubated at $24^{\circ} \mathrm{C}$ in the dark. The readings were taken at $590 \mathrm{~nm}$ after inoculation and at $12 \mathrm{~h}$ intervals for $168 \mathrm{~h}$ using a microplate reader (Victor ${ }^{\mathrm{TM}}$ X5 Multilabel Plate Readers, PerkinElmer). The absorbance measurements for individual substrates were corrected as described by Azarbad et al. (2013). The corrected absorbance values were used to calculate the following microbial indices: substrate richness (Rs), functional diversity based on the Shannon-Wiener biodiversity index (H') and evenness (I') (Klimek and Niklińska 2007). The calculated indices were used in statistical analyses.

\section{Statistical analysis}

Statistical analysis was performed using STATISTICA 10.0 PL software (StatSoft, Tulsa, USA). Differences among samples were analysed by one-way analysis of variance (ANOVA), followed by a post hoc least significant difference test in order to discriminate among the means. The Pearson's correlation coefficients of the data were calculated to determine the relationships among the parameters at the significance levels $\mathrm{p}<0.05$ and $\mathrm{p}<0.01$. The physicochemical and biological results were analysed by means of PCA performed on the correlation matrix of the values of area under the color development profile.

\section{Results and discussion}

\section{Soils contamination with PAHs and metals}

The total concentrations of PAHs in analysed soils varied from 171 to $2137 \mathrm{mg} \mathrm{kg}^{-1}$ dry soil weight (Table 2). The results of PAHs content in tested soils indicated that the contamination was not only unevenly distributed across the site but also very high. The most contaminated soil II exhibited extremely high concentration of naphthalene and the highest level of organic matter (Table 2, 3). This study revealed a particularly strong link $(\mathrm{r}=0.99, \mathrm{p}<0.01)$ between the $\mathrm{OM}$ and naphthalene (Table 4). This fact is in agreement with the findings of Cai et al. (2007) and Nam et al. (2008), who suggest that low molecular weight (LMW) PAHs (with 2 or 3 benzene rings) might more easily approach equilibrium with soil OM over a larger spatial scale (Cai et al. 2007, Nam et al. 2008). Moreover, it has been mentioned that the determination of OM may be overestimated due to the inclusions of hydrocarbons bound tightly to soil particles (Cai et al. 2007, Nam et al. 2008). In the present study, we observed that the high molecular weight (HMW) compounds (with 4 or more benzene rings) were the predominant PAHs found in soils I, III and IV. In the case of soil II they accounted for $47 \%$ of all hydrocarbons. The high level of HMW PAHs in comparison with LMW in soils may result from the different physicochemical properties of these hydrocarbon compounds. The HMW PAHs bind more easily to the soil particles and are less water-soluble thereby these pollutants become less bioavailable and biodegradable by microorganisms (Johnsen and Karlson 2007, Lladó et al. 2009). Total concentrations of seven carcinogenic PAHs (Table 2) varied between $21 \%$ and $36 \%$ and were much lower than previously reported (50\% $\Sigma 16 \mathrm{WWA})$ in the Silesia area (Maliszewska-Kordybach et al. 2009).

The presence of heavy metals was also observed in the tested soils (Table 3). Soils III and IV had the highest amount of $\mathrm{Pb}, \mathrm{Ni}$ and $\mathrm{Cu}$ whose concentrations reached the values of $3378,519,143$ and $3478,664,169 \mathrm{mg} \mathrm{kg}^{-1}$ dry soil, respectively. In most soil samples, the concentrations of lead and nickel exceeded the Polish regulation guidelines (Dz.U. 2002). It is well known that the bioavailability of metals is dependent on $\mathrm{pH}$ (Vig et al. 2003). In the tested soils, the $\mathrm{pH}$ was neutral or slightly alkaline (Table 3 ) which indicates that heavy metals were most likely in an inaccessible form and hence did not affect the microbial communities (Ross 1994, Antoniadis et al. 2008). This is supported by the observation that no negative correlation between enzymatic activity and heavy metals content was noted (Table 4). In our study a strong positive correlation $(\mathrm{p}<0.01)$ between the sum of 16 PAHs and cadmium $(r=0.74)$ and zinc $(\mathrm{r}=0.82)$ was observed (Table 4$)$, pointing to emission from common sources. Surprisingly, there were no statistically significant correlations between PAHs and other metals: $\mathrm{Pb}$, $\mathrm{Cu}$ and $\mathrm{Ni}$. This fact is difficult to explain, and may suggest other source of contamination than coke production, especially since these metals have been strongly correlated to each other. Nevertheless, PAHs and heavy metals in long-term contaminated soils could have a strong negative impact on microbial diversity and activity (Maliszewska-Kordybach et al. 2009, Thavamani et al. 2012, Pessacq et al. 2015). 
Table 1. The limit of detection and recovery values of tested parameters

\begin{tabular}{|c|c|c|}
\hline Parameter & $\begin{array}{c}\text { Limit of detection } \\
{\left[\mathrm{mg} \mathrm{kg}^{-1}\right]}\end{array}$ & $\begin{array}{c}\text { Recovery } \\
{[\%]}\end{array}$ \\
\hline $\mathrm{Cd}$ & 0.87 & 84.8 \\
\hline $\mathrm{Pb}$ & 3.39 & 95.7 \\
\hline $\mathrm{Zn}$ & 8.73 & 98.8 \\
\hline $\mathrm{Cu}$ & 4.34 & 95.0 \\
\hline $\mathrm{Ni}$ & 4.16 & 95.3 \\
\hline Naphthalene & 0.066 & $>70.0$ \\
\hline Acenaphthene & 0.01 & $>70.0$ \\
\hline Fluorene & 0.003 & $>70.0$ \\
\hline Phenanthrene & 0.008 & $>70.0$ \\
\hline Anthracene & 0.002 & $>70.0$ \\
\hline Fluoranthene & 0.016 & 96.1 \\
\hline Pyrene & 0.011 & 95.4 \\
\hline Benzo(a)anthracene & 0.006 & 93.3 \\
\hline Chrysene & 0.006 & 95.3 \\
\hline Benzo(b)fluoranthene & 0.007 & 94.4 \\
\hline Benzo(k)fluoranthene & 0.003 & 93.2 \\
\hline Benzo(a)pyrene & 0.004 & 88.8 \\
\hline Benzo(ghi)perylene & 0.004 & 90.4 \\
\hline Dibenzo(ah)anthracene & 0.002 & 92.6 \\
\hline Indeno(1,2,3-cd)pyrene & 0.004 & 9.3 \\
\hline & & \\
\hline & & 9.3 \\
\hline
\end{tabular}

Table 2. The concentrations of individual and total PAHs in tested soils ${ }^{a}$

\begin{tabular}{|c|c|c|c|c|}
\hline \multirow{2}{*}{ PAHs [mg kg-1] } & \multicolumn{4}{|c|}{ SOILS } \\
\hline & I & II & III & IV \\
\hline Naphthalene & $24.6 \pm 4.1$ & $476.6 \pm 78.6$ & $14.0 \pm 2.3$ & $37.8 \pm 6.2$ \\
\hline Acenaphthene & $8.2 \pm 1.1$ & $54.1 \pm 7.3$ & $5.3 \pm 0.7$ & $4.2 \pm 0.6$ \\
\hline Fluorene & $4.9 \pm 0.6$ & $85.6 \pm 11.1$ & $20.9 \pm 2.7$ & $21.7 \pm 2.8$ \\
\hline Phenanthrene & $25.0 \pm 2.5$ & $392.8 \pm 39.3$ & $92.5 \pm 9.3$ & $95.3 \pm 9.5$ \\
\hline Anthracene & $7.7 \pm 0.7$ & $119.7 \pm 11.4$ & $21.3 \pm 2.0$ & $18.1 \pm 1.7$ \\
\hline Fluoranthene & $29.9 \pm 3.0$ & $322.0 \pm 32.2$ & $78.0 \pm 7.8$ & $166.8 \pm 16.7$ \\
\hline Pyrene & $18.0 \pm 1.8$ & $189.3 \pm 18.9$ & $58.3 \pm 5.8$ & $63.6 \pm 6.4$ \\
\hline Benzo(a)anthracene* & $10.4 \pm 0.8$ & $101.5 \pm 8.1$ & $36.9 \pm 3.0$ & $37.6 \pm 3.0$ \\
\hline Chrysene* $^{*}$ & $6.8 \pm 0.6$ & $68.5 \pm 5.5$ & $37.5 \pm 3.0$ & $25.7 \pm 2.1$ \\
\hline Benzo(b)fluoranthene* & $7.3 \pm 0.7$ & $62.9 \pm 6.3$ & $25.2 \pm 2.5$ & $24.5 \pm 2.5$ \\
\hline Benzo(k)fluoranthene ${ }^{*}$ & $3.9 \pm 0.3$ & $37.1 \pm 3.2$ & $13.4 \pm 1.1$ & $12.7 \pm 1.1$ \\
\hline Benzo(a)pyrene* & $9.7 \pm 0.8$ & $88.2 \pm 7.1$ & $34.3 \pm 2.7$ & $32.9 \pm 2.6$ \\
\hline Benzo(ghi)perylene & $4.4 \pm 0.4$ & $42.1 \pm 3.8$ & $14.1 \pm 1.3$ & $18.3 \pm 1.6$ \\
\hline Dibenzo(ah)anthracene* & $2.2 \pm 0.2$ & $20.5 \pm 1.7$ & $1.2 \pm 0.1$ & $4.2 \pm 0.4$ \\
\hline Indeno(1,2,3-cd)pyrene* & $8.3 \pm 0.8$ & $76.0 \pm 6.8$ & $24.2 \pm 2.2$ & $31.8 \pm 2.9$ \\
\hline Acenaphthylene & - & - & $4.7 \pm 0.5$ & $9.4 \pm 1.0$ \\
\hline$\Sigma 16 \mathrm{PAHs}$ & 171.4 & 2137.1 & 481.6 & 604.6 \\
\hline
\end{tabular}


Table 3. The physical and chemical properties of tested soils ${ }^{a}$

\begin{tabular}{|c|c|c|c|c|}
\hline \multirow{2}{*}{ Parameter } & \multicolumn{4}{|c|}{ SOILS } \\
\hline & 1 & II & III & IV \\
\hline Sand \% & 95 & 80 & 98 & 96 \\
\hline Silt \% & 5 & 2 & 2 & 4 \\
\hline Clay \% & 0 & 18 & 0 & 0 \\
\hline $\mathrm{pH}$ & $7.2 \pm 0.1$ & $8.3 \pm 0.2$ & $7.5 \pm 0.1$ & $7.3 \pm 0.1$ \\
\hline Moisture \% & $20.1 \pm 1.0$ & $21.7 \pm 1.1$ & $14.1 \pm 0.7$ & $13.4 \pm 0.7$ \\
\hline OM \% & $2.19 \pm 0.12$ & $8.79 \pm 0.81$ & $1.95 \pm 0.53$ & $2.25 \pm 0.3$ \\
\hline Total N \% & $5.3 \pm 0.1$ & $0.09 \pm 0.00$ & $0.1 \pm 0.00$ & $0.17 \pm 0.00$ \\
\hline Total P \% & $0.01 \pm 0.00$ & $0.01 \pm 0.00$ & $1.24 \pm 0.03$ & $1.16 \pm 0.03$ \\
\hline Conductivity $\mu \mathrm{S} \mathrm{cm}^{-1}$ & $367.3 \pm 18.4$ & $214.7 \pm 10.7$ & $95.0 \pm 4.8$ & $248.1 \pm 12.4$ \\
\hline $\mathrm{Cd} \mathrm{mg} \mathrm{kg}^{-1}$ soil & $2.6 \pm 0.4$ & $3.1 \pm 0.5$ & $3.1 \pm 0.5$ & $2.8 \pm 0.5$ \\
\hline $\mathrm{Pb} \mathrm{mg} \mathrm{kg}{ }^{-1}$ soil & $57.8 \pm 2.9$ & $1843 \pm 92.2$ & $3378 \pm 168.9$ & $3478 \pm 173.9$ \\
\hline Zn mg kg-1 soil & $247 \pm 12.4$ & $704 \pm 35.2$ & $556 \pm 27.8$ & $504 \pm 25.2$ \\
\hline Cu mg kg ${ }^{-1}$ soil & $11.8 \pm 0.5$ & $99.7 \pm 4.0$ & $143.8 \pm 5.8$ & $169.8 \pm 6.8$ \\
\hline Ni mg kg-1 soil & $10.5 \pm 0.4$ & $184.2 \pm 7.4$ & $519.7 \pm 20.8$ & $666.4 \pm 26.7$ \\
\hline
\end{tabular}

a Data are presented as average \pm standard deviation, $\mathrm{n}=3$.

\section{Enzyme activity}

The activity of soil enzymes is often used to monitor the effects of various pollutants on microbial functioning (Turgay et al. 2010, Tang et al. 2014, Tomkiel et al. 2015). In the tested soils, the activities of a range of enzymes contributing to the biogeochemical cycle of carbon, nitrogen and phosphorus were determined (Fig. 1A-C). In addition, the activity of dehydrogenase, present only in viable cells was assessed. DHA is linked to microbial respiration and reflects the ability of microorganisms to degrade hydrocarbons (Achuba and Okoh 2014, Wang et al. 2014). In the tested soils, despite the presence of strong PAHs and metal co-contamination, a significant activity of the investigated enzymes was observed. The most contaminated soil exhibited a particularly high DHA as well as significant acid and alkaline phosphatase activity (Fig. 1A-B). Moreover, we observed strong positive correlations $(p<0.01)$ between the activity of these enzymes and the total PAHs (Table 4). Several studies have demonstrated that DHA is among the most sensitive parameters for evaluation of hydrocarbons toxicity and stress (Irha et al. 2003, Gianfreda et al. 2005). Turgay et al. (2010) observed that the decrease in the content of hydrocarbons resulted in an increase of DHA over time during the bioremediation process of soil contaminated with petroleum hydrocarbons. However, Tang et al. (2012) examining the bioavailability of petroleum hydrocarbons under different attenuation conditions noticed an increase in DHA immediately after the contamination of soil and its decline with the duration of the experiment. Observed in our study high dehydrogenase and other enzyme activities in the most contaminated soil suggested significant intensification of microbial processes. This phenomenon may be a microbial response to a constant stress exerted by contaminants. Moreover, such results may be related to the high level of organic matter and moisture found in the most contaminated soil II. The highest urease activity and the lowest dehydrogenase and acid phosphatase activities were found in soil IV. Soil I, which was the least contaminated one, had the lowest activities of urease and alkaline phosphatase. Contrary to some other studies (Gianfreda et al. 2005, Turgay et al. 2010, Wu et al. 2014) no correlation was observed between urease activity and the level of PAHs. Thus, the variations in enzyme activities between soil samples showed that in tested soils pollution inputs have specific site effects, which may be a result of lower sensitivity of local microbial communities to PAHs and heavy metals. For instance, Qasemian et al. (2012) found an increased activity of acid phosphatase, cellulase and $\beta$-glucosidase in the leaf litter of forest pine (Pinus halepensis) after 3 months of anthracene contamination. The changes in the activity of an individual enzyme did not allow for a precise quantification of soil degradation by long-time mixed contamination. This finding demonstrates that microorganisms still function when subjected to high levels of pollution.

\section{Catabolic diversity of bacterial communities}

The potential activity of bacterial communities that were able to grow on the substrate provided was measured using Biolog method. Very low metabolic activity with respect to 31 substrates in communities from soils I and II indicated the microbial activity at a basal metabolic rate. It is remarkable that soil III has shown the highest value of average well-color development (AWCD) which expressed the catabolic activity of metabolic communities on EcoPlates ${ }^{\mathrm{TM}}$ but a low enzyme activity. The low activity of tested enzymes may be related to the inhibition effects caused by toxic hydrocarbons. Moreover, soil enzyme activity provides information about the breaking of different chemical bonds whereas the Biolog method reflects the metabolic activity of soil bacteria in response to 31 carbon sources, which were present at high concentrations (Palmroth et al. 2005). Furthermore, CLPPs do not measure the functional abilities of the entire soil microbial community but only that of a very limited subset of microbial fraction capable to grow 


\begin{tabular}{|c|c|c|c|c|c|c|c|c|c|c|c|c|c|c|c|c|c|c|c|c|c|c|c|}
\hline$\frac{0}{0}$ & & & & & & & & & & & & & & & & & & & & \begin{tabular}{l}
$*$ \\
\multirow{2}{0}{} \\
8 \\
0
\end{tabular} & 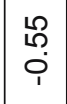 & is & 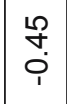 \\
\hline $\begin{array}{l}\Phi \\
\mathscr{D} \\
\mathbb{\Phi} \\
\stackrel{Ð}{J}\end{array}$ & & & & & & & & & & & & & & & & & & & 占 & $\begin{array}{l}N \\
\stackrel{1}{0} \\
i\end{array}$ & 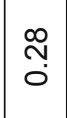 & 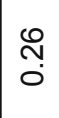 & ָ̃ \\
\hline 좀 & & & & & & & & & & & & & & & & & & $\begin{array}{l}\text { शู } \\
\text { î }\end{array}$ & 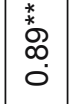 & $\mid$\begin{tabular}{l}
$*$ \\
\multirow{2}{*}{} \\
0 \\
0 \\
0
\end{tabular} & 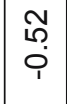 & 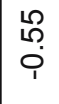 & 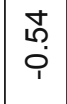 \\
\hline$\frac{T}{S}$ & & & & & & & & & & & & & & & & & 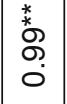 & ז́ & 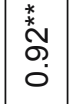 & $\mid \begin{array}{l}* \\
* \\
\infty \\
0 \\
0\end{array}$ & $\begin{array}{l}0 \\
0 \\
0 \\
0 \\
i \\
\end{array}$ & $\begin{array}{l}* \\
0 \\
0 \\
0 \\
0 \\
1\end{array}$ & \begin{tabular}{|l|} 
\\
0 \\
0 \\
0 \\
0 \\
$i$ \\
\end{tabular} \\
\hline 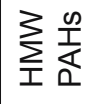 & & & & & & & & & & & & & & & & 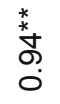 & $\mid$\begin{tabular}{l}
$*$ \\
\multirow{2}{*}{} \\
0 \\
0 \\
0
\end{tabular} & $\stackrel{n}{\circ}$ & \begin{tabular}{|c|}
$*$ \\
\multirow{2}{*}{} \\
$\infty$ \\
0 \\
0
\end{tabular} & $\mid \begin{array}{c}* \\
* \\
\infty \\
\infty \\
0 \\
0\end{array}$ & $\begin{array}{l}m \\
m \\
0 \\
0\end{array}$ & $\begin{array}{c}\hat{m} \\
0 \\
0\end{array}$ & $\begin{array}{c}\tilde{m} \\
\text { o. } \\
0 \\
\end{array}$ \\
\hline$\stackrel{0}{\frac{\infty}{1}} \frac{1}{\alpha}$ & & & & & & & & & & & & & & & \begin{tabular}{l}
$*$ \\
\multirow{2}{*}{} \\
8 \\
0 \\
0
\end{tabular} & 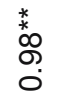 & \begin{tabular}{|l|}
$*$ \\
6 \\
$O$ \\
0 \\
0
\end{tabular} & ח̊ & \begin{tabular}{l}
$*$ \\
\multirow{2}{*}{} \\
0 \\
0 \\
0
\end{tabular} & $\mid \begin{array}{l}* \\
* \\
\infty \\
0 \\
0\end{array}$ & 氶 & $\begin{array}{l}0 \\
0 \\
0 \\
1\end{array}$ & $\begin{array}{l}0 \\
0 \\
0 \\
1\end{array}$ \\
\hline $\bar{z}$ & & & & & & & & & & & & & & $\begin{array}{l}0 \\
\stackrel{0}{0} \\
i\end{array}$ & $\begin{array}{l}0 \\
0 \\
0 \\
i\end{array}$ & $\begin{array}{l}\infty \\
\dddot{m} \\
0 \\
i\end{array}$ & $\mid \begin{array}{c}\infty \\
\dddot{m} \\
i \\
i \\
1\end{array}$ & \begin{tabular}{|l}
$*$ \\
\multirow{2}{*}{} \\
0 \\
0 \\
0 \\
0
\end{tabular} & m & $\begin{array}{c}\bar{E} \\
\dot{0} \\
1\end{array}$ & 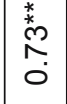 & 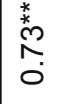 & \begin{tabular}{|l|}
$*$ \\
0 \\
0 \\
0 \\
0 \\
0
\end{tabular} \\
\hline $\mathcal{U}$ & & & & & & & & & & & & & מُ & $\frac{\nabla}{0}$ & 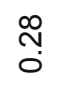 & $\begin{array}{l}0 \\
0 \\
0 \\
0 \\
1\end{array}$ & \begin{tabular}{l|}
0 \\
0 \\
0 \\
$i$
\end{tabular} & 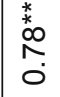 & 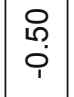 & $\stackrel{0}{0}$ & \begin{tabular}{|l|}
$*$ \\
0 \\
0 \\
0 \\
0
\end{tabular} & $\begin{array}{l}* \\
0 \\
0 \\
0 \\
0\end{array}$ & \begin{tabular}{|l|}
$*$ \\
0 \\
0 \\
0 \\
0 \\
0
\end{tabular} \\
\hline$\tilde{N}$ & & & & & & & & & & & 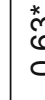 & s̀ & s. & $\begin{array}{c}\underbrace{*}_{*} \\
\vdots \\
0 \\
0 \\
0\end{array}$ & $\mid \begin{array}{l}* \\
0 \\
\infty \\
\infty \\
0 \\
0\end{array}$ & \begin{tabular}{l}
$*$ \\
\multirow{2}{*}{} \\
0 \\
0 \\
0
\end{tabular} & \begin{tabular}{|c|} 
\\
0 \\
0 \\
0 \\
0 \\
0
\end{tabular} & 尺् & \begin{tabular}{|l|}
$*$ \\
0 \\
0 \\
0 \\
0 \\
0
\end{tabular} & $\mid \begin{array}{c}* \\
0 \\
0 \\
0 \\
0\end{array}$ & $\stackrel{N}{\check{O}}$ & $\frac{0}{0}$ & $\begin{array}{l}\infty \\
0 \\
0 \\
\end{array}$ \\
\hline$\frac{0}{\alpha}$ & & & & & & & & & & $\begin{array}{l}* \\
0 \\
0 \\
0 \\
0\end{array}$ & \begin{tabular}{l}
$*$ \\
\multirow{2}{*}{} \\
0 \\
0 \\
$c$
\end{tabular} & . & 'ִ & 용 & $\frac{0}{0}$ & $\begin{array}{l} \pm \\
\dot{0} \\
i\end{array}$ & 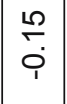 & $\frac{*}{*}$ & ㄲ. & $\stackrel{m}{0}$ & 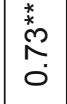 & 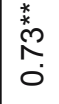 & \begin{tabular}{|l|}
$*$ \\
0 \\
0 \\
0 \\
0 \\
0
\end{tabular} \\
\hline ర & & & & & & & & & $\begin{array}{l}0 \\
1 \\
0 \\
0\end{array}$ & $\begin{array}{l}* \\
6 \\
6 \\
0 \\
0\end{array}$ & $\begin{array}{c}15 \\
15 \\
c\end{array}$ & ๙ & p. & 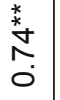 & $\begin{array}{l}* \\
\cdots \\
0 \\
\stackrel{0}{0} \\
0\end{array}$ & $\begin{array}{l}* \\
\cdots \\
0 \\
0 \\
0\end{array}$ & \begin{tabular}{|l|}
\multirow{L}{*}{} \\
$\stackrel{0}{0}$ \\
0 \\
0
\end{tabular} & $\check{\sigma}$ & 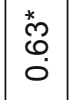 & \begin{tabular}{|l|}
$*$ \\
0 \\
0 \\
0 \\
0
\end{tabular} & $\stackrel{\mathbb{N}}{\mathbb{O}}$ & $\stackrel{\widetilde{n}}{0}$ & 尺ָ̣ \\
\hline 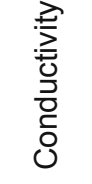 & & & & & & & & 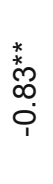 & \begin{tabular}{|l}
$*$ \\
0 \\
0 \\
0 \\
0 \\
0 \\
1
\end{tabular} & \begin{tabular}{l}
$*$ \\
\multirow{2}{*}{} \\
$\stackrel{1}{1}$
\end{tabular} & م̂ & ${ }^{*}$ & 'ִ & $\begin{array}{l}\text { N̦ } \\
\text { î }\end{array}$ & $\begin{array}{l}\bar{m} \\
\text { ọ }\end{array}$ & $\begin{array}{l}\infty \\
0 \\
0 \\
0 \\
1\end{array}$ & $\begin{array}{l}\stackrel{N}{0} \\
\dot{0} \\
i\end{array}$ & 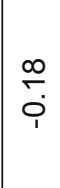 & $\begin{array}{l}\stackrel{\nabla}{+} \\
\dot{1} \\
\end{array}$ & $\mid \begin{array}{l}0 \\
\stackrel{2}{0} \\
\stackrel{1}{1}\end{array}$ & 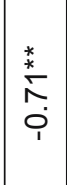 & 莡 & \begin{tabular}{|l|} 
\\
0 \\
0 \\
0 \\
0 \\
1
\end{tabular} \\
\hline $\begin{array}{l}\frac{a}{\pi} \\
\frac{\pi}{0} \\
\frac{0}{0}\end{array}$ & & & & & & & ' & $\stackrel{9}{\circ}$ & \begin{tabular}{|l|}
$*$ \\
$*$ \\
0 \\
0 \\
0 \\
0
\end{tabular} & $\hat{c}$ & $\stackrel{*}{*} \underset{\alpha}{\alpha}$ & $\overbrace{0}^{*}$ & مُ & 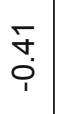 & $\begin{array}{l}\infty \\
\stackrel{\infty}{0} \\
i \\
i\end{array}$ & $\begin{array}{l}1 \\
10 \\
0 \\
1\end{array}$ & \begin{tabular}{|l|}
$*$ \\
$\infty$ \\
0 \\
0 \\
0 \\
1
\end{tabular} & $\begin{array}{l}* \\
6 \\
0 \\
0\end{array}$ & 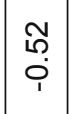 & $\begin{array}{c}\stackrel{2}{0} \\
0 \\
1 \\
1\end{array}$ & \begin{tabular}{|l|}
$*$ \\
$*$ \\
0 \\
$\infty$ \\
0 \\
0 \\
0
\end{tabular} & 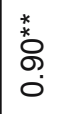 & 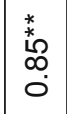 \\
\hline $\begin{array}{l}z \\
\frac{\pi}{0} \\
\stackrel{0}{0}\end{array}$ & & & & & $\begin{array}{l}5 \\
15 \\
1 \\
1\end{array}$ & & : & 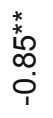 & \begin{tabular}{|l}
$*$ \\
$*$ \\
0 \\
$\infty$ \\
0 \\
0
\end{tabular} & $\begin{array}{l}* \\
* \\
* \\
c \\
c\end{array}$ & 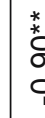 & \begin{tabular}{l}
$*$ \\
\multirow{2}{*}{} \\
$\stackrel{c}{c}$
\end{tabular} & مُ & 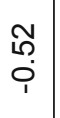 & $\begin{array}{l}* \\
0 \\
0 \\
0 \\
i \\
1\end{array}$ & $\begin{array}{l}\tilde{j} \\
\text { i. } \\
i\end{array}$ & \begin{tabular}{|l|}
$m$ \\
$m$ \\
0 \\
$i$ \\
1
\end{tabular} & $\begin{array}{l}* \\
0 \\
0 \\
0 \\
0 \\
0 \\
i\end{array}$ & 勇 & $\mid \begin{array}{l}0 \\
\tilde{S} \\
i \\
i\end{array}$ & $\begin{array}{l}8 \\
0 \\
0 \\
1\end{array}$ & 尔 & $\begin{array}{l}0 \\
\\
0 \\
i \\
i\end{array}$ \\
\hline$\sum_{0}$ & & & & $\begin{array}{l}\tilde{m} \\
\text { m } \\
\text { i }\end{array}$ & 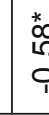 & & $\begin{array}{l}0 \\
0 \\
0 \\
1\end{array}$ & $\begin{array}{l}* \\
\overleftarrow{6} \\
0\end{array}$ & مإم & $\begin{array}{l}x_{0}^{\infty} \\
0 \\
0 \\
c\end{array}$ & c & c & 'ִ & \begin{tabular}{l} 
t. \\
\multirow{2}{*}{} \\
$\stackrel{0}{0}$ \\
0
\end{tabular} & 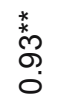 & \begin{tabular}{l} 
* \\
\multirow{2}{*}{} \\
$\stackrel{2}{0}$ \\
0
\end{tabular} & \begin{tabular}{|l|} 
\\
$*$ \\
$o$ \\
$o$ \\
0 \\
0
\end{tabular} & $\stackrel{m}{\stackrel{m}{0}}$ & 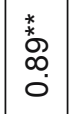 & \begin{tabular}{l|} 
\\
$*$ \\
0 \\
0 \\
0 \\
0
\end{tabular} & $\begin{array}{c}\mathbf{0} \\
0 \\
0 \\
0 \\
1\end{array}$ & $\begin{array}{l}* \\
0 \\
0 \\
0 \\
0 \\
0 \\
1\end{array}$ & $\begin{array}{l}\tilde{b} \\
0 \\
0 \\
1\end{array}$ \\
\hline $\begin{array}{l}\stackrel{0}{\underline{2}} \\
\frac{.00}{0.0} \\
\frac{0}{0}\end{array}$ & & & $\stackrel{\text { I }}{\text { S. }}$ & ¿ & $\begin{array}{l}* \\
0 \\
0 \\
0 \\
1 \\
1 \\
1\end{array}$ & & . & $\begin{array}{l}\stackrel{L}{n} \\
\stackrel{1}{1}\end{array}$ & $\begin{array}{l}x \\
b \\
0 \\
0 \\
0 \\
0\end{array}$ & \begin{tabular}{l}
$c$ \\
\multirow{2}{*}{} \\
1
\end{tabular} & 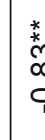 & 年 & 'ִ & $\begin{array}{c}\stackrel{2}{N} \\
0\end{array}$ & $\stackrel{N}{\leftarrow}$ & 守 & 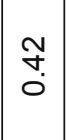 & $\mid$ & 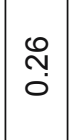 & ơ & $\begin{array}{l}x_{0}^{*} \\
0 \\
0 \\
0 \\
0 \\
\end{array}$ & 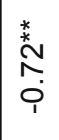 & $\begin{array}{l}* \\
0 \\
0 \\
0 \\
0 \\
1\end{array}$ \\
\hline$\frac{T}{Q}$ & & $\begin{array}{l}\stackrel{N}{0} \\
\text { o }\end{array}$ & 悉 & $\begin{array}{l}5 \\
\text { in } \\
i\end{array}$ & $\begin{array}{l}q \\
\text { f } \\
\end{array}$ & & '. & 悉 & $\begin{array}{l}t \\
0\end{array}$ & 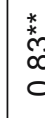 & c & ¿̇ & דיר & 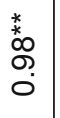 & \begin{tabular}{l}
$*$ \\
\multirow{*}{*}{} \\
$\mathscr{O}$ \\
0 \\
0
\end{tabular} & \begin{tabular}{l}
$*$ \\
\multirow{*}{*}{} \\
$\mathscr{O}$ \\
0 \\
0
\end{tabular} & \begin{tabular}{|l|} 
\\
\multirow{2}{*}{} \\
$o$ \\
0 \\
0
\end{tabular} & 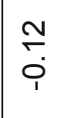 & $\begin{array}{l}* \\
* \\
\sigma \\
0 \\
0\end{array}$ & \begin{tabular}{|c|}
$*$ \\
$*$ \\
$\infty$ \\
$\infty$ \\
0 \\
0
\end{tabular} & 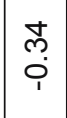 & $\begin{array}{c}\hat{m} \\
0 \\
i\end{array}$ & \begin{tabular}{|c}
$\hat{0}$ \\
0 \\
$i$ \\
\end{tabular} \\
\hline ర) & $\frac{T}{Q}$ & $\begin{array}{l}\frac{0}{3} \\
\frac{0}{00} \\
\frac{0}{2}\end{array}$ & $\sum$ & $\frac{z}{\frac{\pi}{\pi}}$ & $\frac{a}{\frac{\pi}{\sigma}}$ & & 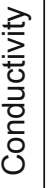 & రె & $\frac{0}{2}$ & ก์ & $\bar{c}$ & $\ddot{z}$ & $\bar{z}$ & $\begin{array}{l}\frac{0}{1} \\
\frac{1}{\Delta} \\
0 \\
\frac{0}{W}\end{array}$ & 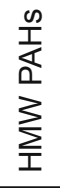 & $\frac{T}{\Sigma}$ & $\begin{array}{l}\mathbb{3} \\
\mathbf{1} \\
\mathbf{a}\end{array}$ & 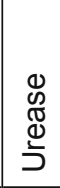 & $\begin{array}{l}\frac{0}{0} \\
0 \\
\end{array}$ & 㕸 & 足 & I & $=$ \\
\hline
\end{tabular}



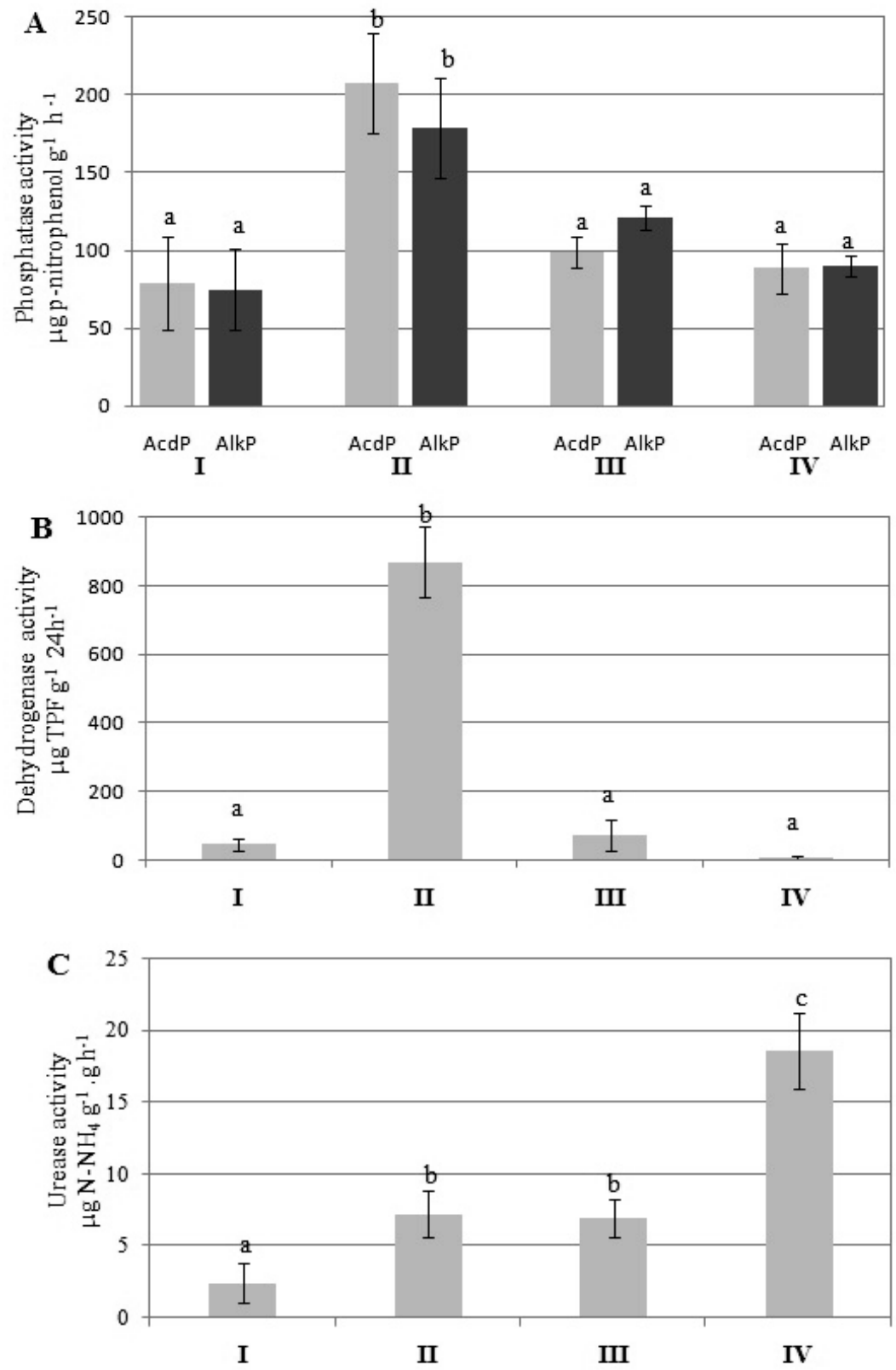

Fig. 1. Acid and alkaline phosphatase (A), dehydrogenase (B) and urease (C) activity in tested soils

on the carbon sources provided (Palmroth et al. 2005, Hueso et al. 2012). To assess the potential functional diversity of the bacterial community H', I' and Rs indices were calculated (Table 5). The functional diversity and evenness indices were the highest in soil III and the lowest in soil I and II. Microbial communities in soil III were characterised by high species richness and an even distribution of functional abilities within the community. Bacterial communities in the most and the least contaminated soils reveal very low functional biodiversity. No correlations were found between the Shannon indices and the sum of 16 PAHs, which is in contradistinction to the findings of Zhang et al. (2010) who reported a correlation between these two factors. However, Muckian et al. (2007) found that the level of PAHs did not alter the number of ribotypes in the contaminated soils. They indicated that the PAH ring structure plays a greater role in influencing the bacterial community than the total PAHs concentration. In our study, a negative correlation between H' and naphthalene, a 2-ring PAH, was observed (Table 4). This can be explained by different properties of these hydrocarbons. Naphthalene is the most soluble among PAHs and thus more mobile and toxic to microorganisms. No correlations were found between functional diversity indices and enzyme activities. The functional diversity of bacterial communities was positively correlated with total phosphorus $(\mathrm{r}=0.90)$, lead $(\mathrm{r}=0.73)$ and nickel content $(\mathrm{r}=0.73, \mathrm{p}<0.01)$. There were also observed strong negative correlations between diversity indices and moisture $(\mathrm{r}=-0.72)$ and conductivity $(\mathrm{r}=-0.72, \mathrm{p}<0.01)$. It seems that some metals and physicochemical factors are more important in controlling the soil bacterial diversity than hydrocarbons (Azarbad et al. 2013). The number of utilized carbon sources, which is defined as the richness index, varied depending on the soil, from 26 to 31 out of 31 carbon sources available on the Biolog EcoPlates $^{\mathrm{TM}}$ (Table 5). Two soils exhibited strongly reduced richness indices: the most contaminated (II) one and the least contaminated (I) one. 
Table 5. Richness (Rs), Shannon-Weaver ( $\left.\mathrm{H}^{\prime}\right)$ and evenness (l') indices calculated on carbon substrate used in Biolog EcoPlate ${ }^{\mathrm{TM}}$

\begin{tabular}{|c|c|c|c|c|}
\hline \multirow{2}{*}{ Index } & \multicolumn{4}{|c|}{ SOILS } \\
\cline { 2 - 5 } & $\mathrm{I}$ & II & III & IV \\
\hline Rs & $27 \pm 1.2 \mathrm{a}$ & $26 \pm 0.6 \mathrm{a}$ & $31 \pm 0.6 \mathrm{~b}$ & $29 \pm 0.6 \mathrm{c}$ \\
\hline $\mathrm{H}^{\prime}$ & $1.30 \pm 0.02 \mathrm{a}$ & $1.28 \pm 0.01 \mathrm{a}$ & $1.44 \pm 0.03 \mathrm{~b}$ & $1.36 \pm 0.01 \mathrm{c}$ \\
\hline I' & $0.91 \pm 0.02 \mathrm{a}$ & $0.90 \pm 0.01 \mathrm{a}$ & $0.97 \pm 0.01 \mathrm{~b}$ & $0.94 \pm 0.01 \mathrm{c}$ \\
\hline
\end{tabular}

Values with the same letter mean indicate that there are no significant differences $(p<0.05)$
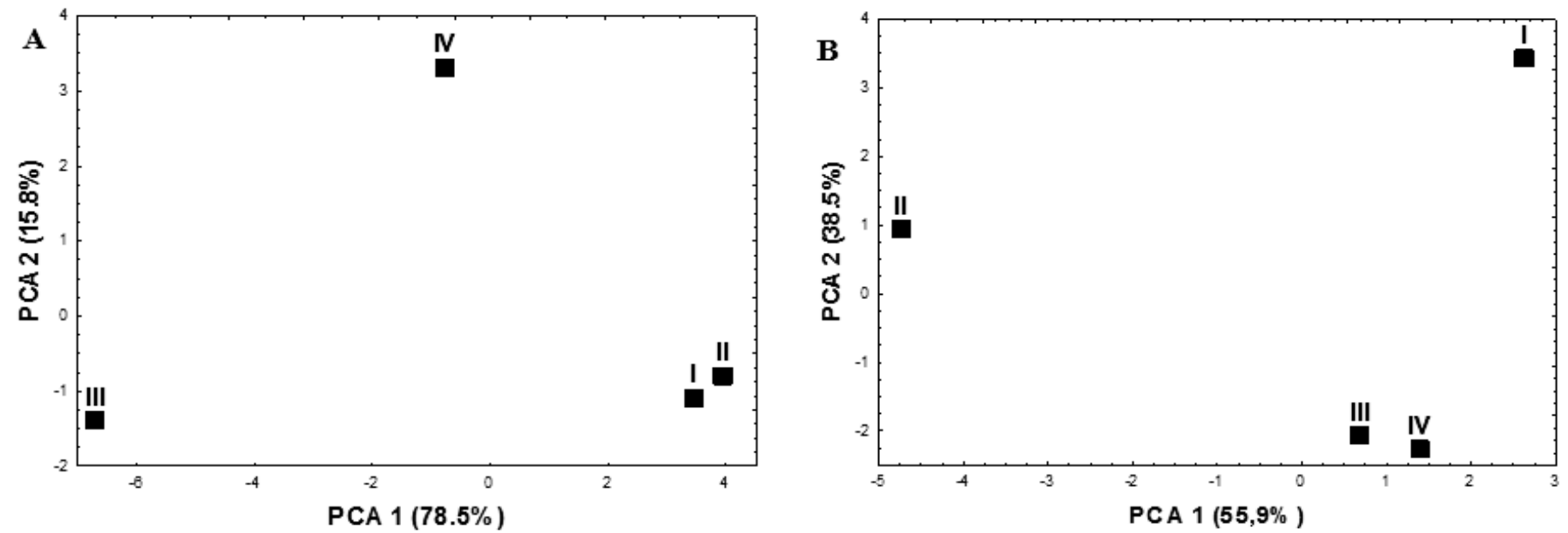

Fig. 2. Principal Component Analysis (PCA) of (A) carbon utilization profiles of bacterial communities (B) environmental factors and enzymatic activities of tested soils. Each point represents the bar center of three replicates

\section{Principal component analysis}

All the obtained data were subjected to principal component analysis (Fig. 2A-B). The first PCA was carried out for carbon utilization profiles. PCA revealed differences in functional diversity between the bacterial communities present in the tested soils. Both the most contaminated (II) and the least contaminated (I) soils exhibited a noticeable separation from the two other soils in the first principal component (PC1), which explained $78.5 \%$ of the variation in the data. The reason for these findings may be the selection and adaptation of microorganisms to the pollutants, which cause a lack of species or strains capable to degrade a variety of carbon sources (Wu et al. 2014). The second PCA analysis was carried out for physicochemical data, $\Sigma 16 \mathrm{PAHs}$ concentrations and enzymatic activities (Fig 2B). The majority of the variance (94.4\%) was explained by the two principal components. Both soils III and IV exhibited a significant separation from the other two soils (I and II) along the first and the second axis. The PC1 was highly negatively correlated with the sum of PAHs, the activity of dehydrogenase, both phosphatases, as well as with $\mathrm{pH}$, whereas the second factor was highly positively correlated with moisture and negatively correlated with total $\mathrm{P}, \mathrm{Pb}, \mathrm{Cu}$ and $\mathrm{Ni}$.

The long-term pressure of the mixture of contaminants may have modified the microbial metabolism and/or the relative composition and size of the constituent populations. This is because microbial communities have the ability to adapt, physiologically and evolutionally, to the presence of contaminants in soil. After the period of "stress", there is an increase in respiration intensity and enzyme activity (Baran et al. 2004, Pessacq et al. 2015). This could have occurred only in the presence of acclimated microbial communities, which were capable of adapting and proliferating in the presence of high levels of PAHs and metals. Understanding these complex interactions is of major importance for the bioremediation of contaminated soils (Thavamani et al. 2012).

\section{Conclusions}

Our study has demonstrated that enzyme activities and microbial functional diversity in PAHs and metal contaminated soils is related and characteristic to a specific site. This fact is connected with the high heterogeneity of soil and uneven distribution of hydrocarbons in soil. The obtained results showed also that the evaluation of a single parameter such as the activity of enzyme does not reflect the real effect of contaminants input on the activity and diversity of microbial communities. Moreover, the results of this study clearly showed that microbial communities have enormous adaptation abilities to the mixed inorganic and organic contamination.

\section{Acknowledgements}

We would like to thank the professional team of Analytical Laboratory in IETU for physical and chemical analysis.

This work was supported by Grant No. N305 051340 financed by the Polish Ministry of Science and Higher Education. 


\section{References}

Achuba, F.I. \& Okoh, P.N. (2014). Effect of petroleum products on soil catalase and dehydrogenase activities, Open Journal of Soil Science, 4, pp. 399-406.

Alef, K. (1995). Dehydrogenase activity, in: Methods in applied soil microbiology and biochemistry, Alef, K. \& Nannipieri, P. (Eds.), Academic Press, London, pp. 228-231.

Antoniadis, V., Robinson, J.S. \& Alloway, B.J. (2008). Effects of short-term $\mathrm{pH}$ fluctuations on cadmium, nickel, lead, and zinc availability to ryegrass in a sewage sludge-amended field, Chemosphere, 71, pp. 759-764.

Azarbad, H., Niklińska, M., van Gestel, C.A.M., van Straalen, N.M., Röling, W.F.M. \& Laskowski, R. (2013). Microbial community structure and functioning along metal pollution gradients, Environmental Toxicology and Chemistry, 32(9), pp. 1992-2002.

Bamforth, S.M. \& Singleton, I. (2005). Bioremediation of polycyclic aromatic hydrocarbons: current knowledge and future directions, Journal of Chemical Technology and Biotechnology, 80, pp. 723-736.

Baran, S., Bielińska, J. \& Oleszczuk, P. (2004). Enzymatic activity in an airfield soil polluted with polycyclic aromatic hydrocarbons, Geoderma, 118, pp. 221-232.

Cai, Q.Y., Mo, C.H., Li, Y.H., Zeng, Q.Y., Katsoyiannis, A., Wu, Q.T. \& Férard, J.F. (2007). Occurrence and assessment of polycyclic aromatic hydrocarbons in soils from vegetable fields of the Pearl River Delta, South China, Chemosphere, 68, pp. 159-168.

Cébron, A., Faure, P., Lorgeoux, C., Ouvrard, S. \& Leyval, C. (2013). Experimental increase in availability of a PAH complex organic contamination from an aged contaminated soil: Consequences on biodegradation, Environmental Pollution, 177, pp. 98-105.

Dz.U. Nr 165, poz. 1359 (2002). Regulation of the Minister of the Environment on the standards of the soil and ground quality (in Polish).

Gianfreda, L., Rao, M.A., Piotrowska, A., Palumbo, G. \& Colombo, C. (2005). Soil enzyme activities as affected by anthropogenic alterations: intensive agricultural practices and organic pollution, Science of the Total Environment, 341, pp. 265-279.

Hueso, S., García, C. \& Hernández, T. (2012). Severe drought conditions modify the microbial community structure, size and activity in amended and unamended soils, Soil Biology and Biochemistry, 50, pp. 167-173.

Irha, N., Slet, J. \& Petersell, V. (2003). Effect of heavy metals and PAH on soil assessed via dehydrogenase assay, Environment International, 28, pp. 779-782.

ISO 10390 (1997). International Standardization Organization. Soil quality - Determination of $\mathrm{pH}$.

ISO 11261 (1995). International Standardization Organization. Soil quality - Determination of total nitrogen - Modified Kjeldahl method.

ISO 11263 (1994) International Standardization Organization. Soil quality - Determination of phosphorus - Spectrometric determination of phosphorus.

ISO 11265 (1997). International Standardization Organization. Soil quality - Determination of conductivity.

ISO 11277 (1998). International Standardization Organization. Soil quality - Determination of particle size distribution in mineral soil material - Method by sieving and sedimentation.

ISO 13877 (1998). Soil quality - Determination of polynuclear aromatic hydrocarbons - Method using high-performance liquid chromatography.

Johnsen, A.R. \& Karlson, U. (2007). Diffuse PAH contamination of surface soils: environmental occurrence, bioavailability, and microbial degradation, Applied Microbiology and Biotechnology, 76, pp. 533-543.
Klimek, B. \& Niklińska, M. (2007). Zinc and copper toxicity to soil bacteria and fungi from zinc polluted and unpolluted soils: A comparative study with different types of Biolog plates, Bulletin of Environmental Contamination and Toxicology, 78, pp. 112-117.

Lladó, S., Jiménez, N., Viñas, M. \& Solanas, A.M. (2009). Microbial populations related to PAH biodegradation in an aged biostimulated creosote-contaminated soil, Biodegradation, 20, pp. 593-601.

Lu, M., Xu, K. \& Chen, J. (2013). Effect of pyrene and cadmium on microbial activity and community structure in soil, Chemosphere, 91, pp. 491-497.

Lu, X-Y., Zhang, T. \& Fang, H.H-P. (2011). Bacteria-mediated PAH degradation in soil and sediment, Applied Microbiology and Biotechnology, 89, pp. 1357-1371.

Maliszewska-Kordybach, B., Smreczak, B. \& Klimkowicz-Pawlas, A. (2009). Effects of anthropopressure and soil properties on the accumulation of polycyclic aromatic hydrocarbons in the upper layer of soils in selected regions of Poland, Applied Geochemistry, 24, pp. 1918-1926.

Muckian, L., Grant, R., Doyle, E. \& Clipson, N. (2007). Bacterial community structure in soils contaminated by polycyclic aromatic hydrocarbons, Chemosphere, 68, pp. 1535-1541.

Nam, J.J., Thomas, G.O., Jaward, F.M., Steinnes, E., Gustafsson, O. \& Jones, K.C. (2008). PAHs in background soils from Western Europe: influence of atmospheric deposition and soil organic matter, Chemosphere, 70, pp. 1596-1602.

Palmroth, M.R.T., Münster, U., Pichtel, J. \& Puhakka, J.A. (2005). Metabolic responses of microbiota to diesel fuel addition in vegetated soil, Biodegradation, 16, pp. 91-101.

Pessacq, J., Medina, R., Terada, C., Bianchini, F.E., Morelli, I.S. \& Del Panno, M.T. (2015). Assessment of the responsiveness to different stresses of the microbial community from long-term hydrocarbon-contaminated soils, Water, Air, \& Soil Pollution, 226, 20. DOI 10.1007/s11270-014-2262-9.

PN-R-04033 (1998). Gleby i utwory mineralne. Podział na frakcje i grupy granulometryczne. Polish Standard. Distribution of the particle sizes in the soil. Polish Committee for Standardization. (in Polish)

Qasemian, L., Guiral, D., Ziarelli, F., Van Dang, T.K. \& Farnet, A.M. (2012). Effects of anthracene on microbial activities and organic matter decomposition in a Pinus halepensis litter from a Mediterranean coastal area, Soil Biology and Biochemistry, 46, pp. 148-154.

Rachwał, M., Magiera, T. \& Wawer, M. (2015). Coke industry and steel metallurgy as the source of soil contamination by technogenic magnetic particles, heavy metals and polycyclic aromatic hydrocarbons, Chemosphere, http://dx.doi.org/10.1016/j. chemosphere.2014.11.077

Ross, S. (1994). Retention, transformation and mobility of toxic metals in soils, in: Toxic metals in soil-plant systems, Ross, S. (Ed.), John Woley Sons, Chichester UK 1994.

Schinner, F., Öhlinger, R., Kandeler, E. \& Margesin, R. (1996). Methods in soil biology. Springer, Heidelberg Berlin, New York 1996.

Tabatabai, M.A. \& Bremner, J.M. (1969). Use of p-nitrophenyl phosphate in assay of soil phosphatase activity, Soil Biology and Biochemistry, 1, pp. 301-307.

Tabatabai, M.A. \& Bremner, J.M. (1972). Assay of urease activity in soils, Soil Biology and Biochemistry, 4, pp. 479-487.

Tang, X., Hashmi, M.Z., Long, D., Chen, L., Khan, M.I. \& Shen, Ch. (2014). Influence of heavy metals and PCBs pollution on the enzyme activity and microbial community of paddy soils around an e-waste recycling workshop, International Journal of Environmental Research and Public Health, 11, pp. 3118-3131. 
Tang, J., Lu, X., Sun, Q. \& Zhu, W. (2012). Aging effect of petroleum hydrocarbons in soil under different attenuation conditions, Agriculture, Ecosystems \& Environment, 149, pp. 109-117.

Thavamani, P., Malik, S., Beer, M., Megharaj, M. \& Naidu, R. (2012). Microbial activity and diversity in long-term mixed contaminated soils with respect to polyaromatic hydrocarbons and heavy metals, Journal of Environmental Management, 99, pp. 10-17.

Tomkiel, M., Baćmaga, M., Wyszkowska, J., Kucharski, J. \& Borowik, A. (2015). The effect of carfentrazone-ethyl on soil microorganisms and soil enzymes activity, Archives of Environmental Protection, 41, pp. 3-10.

Turgay, O.C., Erdogan, E.E. \& Karaca, A. (2010). Effect of humic deposit (leonardite) on degradation of semi-volatile and heavy hydrocarbons and soil quality in crude-oil-contaminated soil, Environmental Monitoring and Assessment, 170, pp. 45-58.

USEPA Method 3620, (1992). (http://www.epa.gov/SW-846/ pdfs/3630c.pdf/ (30.10.2014))

USEPA Method 8440, (1995). (http://www.epa.gov/SW-846/ pdfs/3630c.pdf/ (30.10.2014))

Veses, O., Mosteo, R., Ormad, M.P. \& Ovelleiro, J.L. (2013). Potential risk assessment of trace elements and PAHs in sediment samples of the Ebro river basin (Spain), Bulletin of Environmental Contamination and Toxicology, 90, pp. 136-142.

Vig, K., Megharaj, M., Sethunathan, N. \& Naidu, R. (2003). Bioavailability and toxicity of cadmium to microorganisms and their activities in soil: a review, Advances in Environmental Research, 8 (1), pp. 121-135.

Wang, Y., Fang, L., Lin, L., Luan, T. \& Tam, N.F.Y. (2014). Effects of low molecular-weight organic acids and dehydrogenase activity in rhizosphere sediments of mangrove plants on phytoremediation of polycyclic aromatic hydrocarbons, Chemosphere, 99, pp. 152-159.

Wang, H.S., Liang, P., Kang, Y.A., Shao, D.D., Zheng, G.J., Wu, S.C., Wong, C.K.C. \& Wong, M.H. (2010). Enrichment of polycyclic aromatic hydrocarbons (PAHs) in mariculture sediments of Hong Kong, Environmental Pollution, 158, pp. 3298-3308.

Wu, B., Lan, T., Lu, D. \& Liu, Z. (2014). Ecological and enzymatic responses to petroleum contamination, Environmental Science: Processes \& Impacts, 16, pp. 1501-1509.

Zhang, W., Wang, H., Zhang, R., Yu, X-Z., Qian, P-Y. \& Wong, M.H. (2010). Bacterial communities in PAH contaminated soils at an electronic-waste processing center in China, Ecotoxicology, 19, pp. 96-104.

\section{Aktywność i bioróżnorodność funkcjonalna zespołów mikroorganizmów w glebach długotrwale skażonych węglowodorami oraz metalami ciężkimi}

Streszczenie: Celem pracy było zbadanie wpływu wieloletniego skażenia gleb metalami ciężkimi oraz wielopierścieniowymi węglowodorami aromatycznymi (WWA) na funkcjonowanie zespołów mikroorganizmów. Do badań pobrano gleby z terenu koksowni, gdzie produkcja koksu trwa od 1884 roku. Stężenia WWA w badanych glebach wahały się w przedziale od 171 do $2137 \mathrm{mg} \mathrm{kg}^{-1}$. Wśród badanych metali zawartość ołowiu wynosiła od 57 do $3478 \mathrm{mg} \mathrm{kg}^{-1}$, niklu od 10 do $666 \mathrm{mg} \mathrm{kg}$, a stężenie cynku od 247 do $704 \mathrm{mg} \mathrm{kg}^{-1}$. W najsilniej skażonej glebie zaobserwowano wysoką aktywność dehydrogenazy, fosfatazy kwaśnej i zasadowej. Wyniki takie mogą wskazywać na adaptację zespołów mikroorganizmów do długoletniego zanieczyszczenia metalami i WWA. Jednocześnie analiza profili fizjologicznych zespołów mikroorganizmów (CLPPs) wykazała obniżenie indeksu bioróżnorodności funkcjonalnej. Zaobserwowano również, że obecność metali ( $\mathrm{Pb}, \mathrm{Ni})$ oraz parametry glebowe takie jak wilgotności i przewodności wywierały istotniejszy wpływ na bioróżnorodność metaboliczną niż WWA. 